\title{
Ultrasound-mediated biophotonic imaging: A review of acousto-optical tomography and photo-acoustic tomography
}

\author{
Lihong V. Wang* \\ Optical Imaging Laboratory, Department of Biomedical Engineering, Texas A\&M University, 3120 TAMU, College \\ Station, TX 77843-3120, USA
}

\begin{abstract}
This article reviews two types of ultrasound-mediated biophotonic imaging-acousto-optical tomography (AOT, also called ultrasound-modulated optical tomography) and photo-acoustic tomography (PAT, also called opto-acoustic or thermoacoustic tomography)-both of which are based on non-ionizing optical and ultrasonic waves. The goal of these technologies is to combine the contrast advantage of the optical properties and the resolution advantage of ultrasound. In these two technologies, the imaging contrast is based primarily on the optical properties of biological tissues, and the imaging resolution is based primarily on the ultrasonic waves that either are provided externally or produced internally, within the biological tissues. In fact, ultrasonic mediation overcomes both the resolution disadvantage of pure optical imaging in thick tissues and the contrast and speckle disadvantages of pure ultrasonic imaging. In our discussion of AOT, the relationship between modulation depth and acoustic amplitude is clarified. Potential clinical applications of ultrasound-mediated biophotonic imaging include early cancer detection, functional imaging, and molecular imaging.
\end{abstract}

\section{Introduction}

\subsection{Motivation for use of optical contrast}

Advances in diagnostic technology dramatically have improved the detection and staging of cancer. However, current detection technologies mostly are geared toward establishing diagnoses of clinically overt cancers. Furthermore, most of the current methods are not suitable for use in screening. Imaging techniques, such as X-ray computerized tomography, ultrasound, and magnetic resonance imaging, are limited in their ability to detect small lesions. In most cases, the detected lesions have grown to at least $1 \mathrm{~cm}$ in diameter, have a mass of one gram or more, and contain at least

\footnotetext{
* Corresponding author: Optical Imaging Laboratory, Department of Biomedical Engineering, Texas A\&M University, 3120 TAMU, College Station, TX 77843-3120, USA. Tel.: +1 979847 9040; Fax: +1 979845 4450; E-mail: LWang@tamu.edu; Web Site: http://oilab. tamu.edu.
}

$10^{8}$ cells, which is estimated to represent about 30 doublings from a single malignant cell. In fact, about twothirds of the natural history of a cancer has occurred by the time the cancer is detected by current methods. In other words, these techniques leave a narrow window of opportunity for detection before the tumor burden reaches the lethal range. For example, endoscopic surveillance may fail to identify malignant changes in the bladder epithelium in the early in-situ stages. Consequently, it is imperative to develop new, non-invasive technologies for early detection of incipient neoplasia.

Optical imaging in biological tissue is highly desirable because of the sensitive contrast information it provides which enables potential early detection of neoplasma. Because the optical properties of biological tissue in the visible and near-infrared region of the electromagnetic spectrum are related to the molecular constituents of tissues and their electronic and/or vibrational structures, they are intrinsically sensitive to tissue abnormalities and functions. Optical properties can be used to quantify the hallmarks of cancer-including an- 
giogenesis, hyper-metabolism, pleomorphism, and invasion into normal adjacent tissue-and potentially can offer early cancer detection. Optical properties include scattering and absorption. Optical scattering properties reveal architectural changes in biological tissue at the cellular and sub-cellular levels, whereas optical absorption properties can be used to quantify angiogenesis and hyper-metabolism. Functional optical imaging is possible because optical information can be used to deduce certain physiological parameters such as the oxygen saturation of hemoglobin and the concentration of hemoglobin. Oxygen saturation of hemoglobin is related closely to the metabolic state of lesions and, hence, is an important diagnostic parameter. However, high-resolution optical imaging for greater than $\sim 1 \mathrm{~mm}$ in imaging depth is a challenge because light is strongly scattered in biological tissue. To meet this challenge, combinations of optical and ultrasonic technologies have been explored, because ultrasonic waves scatter much less in biological tissue than do optical waves and can furnish localization information for imaging.

\subsection{Motivation for ultrasound-mediated biophotonic tomography}

This article reviews ultrasound-mediated biophotonic imaging, including acousto-optical tomography (AOT, also called ultrasound-modulated optical tomography) and photo-acoustic tomography (PAT, also called opto-acoustic or thermo-acoustic tomography). In these two technologies, the imaging contrast is based primarily on the optical properties of biological tissues, and the imaging resolution is based primarily on the ultrasonic waves that either are provided externally or produced internally, within the biological tissues. The goal of this type of technology is to combine the contrast advantage inherent in optical properties and the resolution advantage of ultrasound.

In AOT, an ultrasonic wave is focused into biological tissue. Any light that is encoded by the ultrasound, including both singly and multiply scattered photons, contributes to the imaging signal. Axial resolution along the acoustic axis can be achieved with ultrasonicfrequency sweeping and subsequent application of the Fourier transformation, whereas lateral resolution can be obtained by focusing the ultrasonic wave.

In PAT, a short-pulsed laser source is used to irradiate the tissue samples. The photo-acoustic waves excited by thermoelastic expansion are measured by wideband ultrasonic transducers around the sample, and the ac- quired photo-acoustic waves then are used to reconstruct the optical absorption distribution.

The motivation to develop AOT and PAT is summarized in Table 1. Pure optical imaging suffers from strong light scattering in biological tissues [1]. As a result, pure optical imaging has either a limited imaging depth, as in optical coherence tomography (OCT), or a limited resolution, as in diffuse optical tomography (DOT). Compared with optical scattering, ultrasound scattering is 2-3 orders of magnitude weaker in biological tissues [2]. Consequently, pure ultrasound imaging can provide good resolution. However, because ultrasound imaging is based on the detection of mechanical properties in biological tissues, it has weak contrast for early stage tumors and cannot image oxygen saturation or concentration of hemoglobin. Either AOT or PAT overcomes the limitations of the existing pure optical or pure ultrasonic imaging modalities by merging the contrast advantage of pure optical imaging with the resolution advantage of pure ultrasound imaging. In these hybrid methods, trast is related to the optical properties of the sample, yet the resolution is not limited by optical diffusion or multiple photon scattering. The imaging resolution, as well as the maximum imaging depth, is scaleable with the ultrasonic frequency.

\section{Acousto-optical tomography: Introduction}

AOT, also called ultrasound-modulated optical technique, is one of the ultrasound-mediated biophotonic imaging modalities. AOT is based on the ultrasonic modulation of coherent laser light in biological tissue. The modulated component of the speckle pattern carries spatial information determined by the ultrasound and can be utilized for tomographic imaging.

Marks et al. [3] investigated the possibility of tagging light with ultrasound. Wang et al. [4] developed AOT and obtained images in tissue phantoms. Leutz and Maret [5] analyzed ultrasonic modulation of light both theoretically and experimentally. Kemple et al. [6] investigated the scale dependence of the ultrasoundmodulated optical signal on the optical thickness of the scattering medium. Leveque et al. [7] developed a parallel speckle detection scheme that uses a sourcesynchronized lock-in technique in which a CCD camera works as a detector array and obtained one-dimensional (1D) images of buried objects in biological tissue. They found that the signal-to-noise ratio (SNR) can be improved greatly by averaging the signals from all of the CCD pixels. Yao and Wang [8] obtained two- 
Table 1

Motivation for acousto-optical tomography (AOT) and photo-acoustic tomography (PAT) For comparison purposes, we chose optical coherence tomography (OCT) and diffuse optical tomography (DOT) as the pure optical imaging modalities and ultrasonography (US) at $6 \mathrm{MHz}$ as the pure ultrasound imaging modality

\begin{tabular}{|c|c|c|c|c|}
\hline \multirow[t]{2}{*}{ Properties } & \multicolumn{3}{|c|}{ Modalities } & \multirow[b]{2}{*}{ AOT/PAT } \\
\hline & OCT & DOT & US & \\
\hline Contrast & Good & Excellent & Poor for early cancers & $\begin{array}{l}\text { Excellent } \\
(=\text { DOT })\end{array}$ \\
\hline Resolution & $\begin{array}{l}\text { Excellent } \\
(\sim 10 \mu \mathrm{m})\end{array}$ & $\begin{array}{l}\text { Poor } \\
(\sim 5 \mathrm{~mm})\end{array}$ & $\begin{array}{l}\text { Excellent \& scalable } \\
(\sim 150 \mu \mathrm{m})\end{array}$ & $\begin{array}{l}\text { Excellent } \\
(=\mathrm{US})\end{array}$ \\
\hline Imaging depth & $\begin{array}{l}\text { Poor } \\
(\sim 1 \mathrm{~mm})\end{array}$ & $\begin{array}{l}\text { Excellent } \\
(\sim 5 \mathrm{~cm})\end{array}$ & $\begin{array}{l}\text { Good \& scalable } \\
(\sim 3 \mathrm{~cm})\end{array}$ & $\begin{array}{l}\text { Good \& scalable } \\
\text { (= US) }\end{array}$ \\
\hline Speckle artifacts & Strong & None & Strong & None \\
\hline $\begin{array}{l}\text { Scattering } \\
\text { coefficient }\end{array}$ & $\begin{array}{l}\text { Strong } \\
(\sim 100 / \mathrm{cm})\end{array}$ & $\begin{array}{l}\text { Strong } \\
(\sim 100 / \mathrm{cm})\end{array}$ & $\begin{array}{l}\text { Weak } \\
(\sim 0.3 / \mathrm{cm})\end{array}$ & \\
\hline
\end{tabular}

dimensional (2D) images of multiple objects buried in biological tissue by using this technique and also demonstrated that ultrasound-modulated diffuse light contributed to the imaging. Li et al. $[9,10]$ extended the parallel detection scheme with additional methods of analysis. However, because of the elongated ultrasonic focal zone, they discovered that the spatial resolution along the ultrasonic axis is not as good as is the lateral resolution in densely scattering biological tissue.

Wang and $\mathrm{Ku}$ [11] developed a technique called frequency-swept (chirped) AOT to achieve controllable spatial resolution along the ultrasonic axis. In this method, one frequency-sweep (chirp) function excited the ultrasonic transducer while another chirp modulated the gain of the optical detector. When the heterodyne signal from the optical detector was Fourier transformed, the signal from a specific spatial location was converted into a specific frequency component. However, this technique was demonstrated only for ballistic imaging when a single-element detector was used because of the limited SNR. Yao et al. [12] extended the frequency-swept modulation technique [11] using parallel detection [7]. The laser and the ultrasonic transducer both were modulated with chirp functions. Imaging along the ultrasonic axis was accomplished by electronically scanning the time delay between these two chirp functions and 2D images of biological tissue with buried objects were obtained. The scalability of the spatial resolution along the acoustic axis using this technique was studied. They also demonstrated-by using an obliquely incident laser beam-that this technique does not depend on purely ballistic photons.

In addition, Lev et al. developed a reflection configuration for AOT [13], which also was explored by Leveque-Fort et al. [14]. Granot et al. [15] and Yao et al. [8] explored the decay of the modulated signal as a function of imaging depth. Lev and Sfez [16] mapped the photon density distribution using AOT. Selb et al. [17] developed non-linear AOT based on second harmonic ultrasonic modulation of light.

Wang $[18,19]$ developed a full theory describing the mechanisms of ultrasonic modulation of coherent laser light in scattering media. The optical phase of scattered light is modulated through the ultrasound-induced displacement of scatterers and variation of the refractive index. Consequently, the speckle pattern formed by the scattered light is modulated by the ultrasound. The relative importance of the two mechanisms depends on the optical scattering mean free path relative to the acoustic wavelength. Sakadzic and Wang [20] recently extended the analytical solution to anisotropic scattering.

\section{Acousto-optical tomography: Theory}

This section summarizes and further interprets the key results noted in Reference [18]. Three possible mechanisms have been identified for the ultrasonic modulation of light in scattering media. The first mechanism is based on ultrasound-induced variations of the optical properties of the media. As an ultrasonic wave propagates in a scattering medium, the medium is compressed and rarified depending on location and time. Variations of density cause the optical properties of the medium-including the absorption coefficient, the scattering coefficient, and the index of refraction-to vary. Accordingly, the detected intensity of light varies with the ultrasonic wave. However, ultrasonic modulation of incoherent light has been too weak to be observed experimentally.

The second mechanism is based on variations of the optical phase in response to ultrasound-induced displacements of scatterers. The displacements of scatter- 
ers, assumed to follow ultrasonic amplitudes, modulate the physical path lengths of light traversing through the ultrasonic field. Multiply scattered light accumulates modulated physical path lengths along its path. Consequently, the intensity of the speckles formed by the multiply scattered light fluctuates with the ultrasonic wave. A theory developed by Leutz and Maret [5] partially modeled this mechanism but is valid only under the condition that the scattering mean free path is much greater than the acoustic wavelength; unfortunately, under this condition, we will show that this second mechanism is dominated by the third one, described below.

The third mechanism is based on variations of the optical phase in response to ultrasonic modulation of the index of refraction. As a result of ultrasonic modulation of the index of refraction, the optical phase between two consecutive scattering events is modulated. As in the second mechanism, multiply scattered light accumulates modulated phases along its path, and the modulated phase causes the intensity of the speckles formed by the multiply scattered light to vary with the ultrasonic wave. This mechanism has never been modeled.

Both the second and the third mechanisms require the use of coherent light. Recently, an analytical model and a Monte Carlo model, based on both of the mechanisms for the ultrasonic modulation of coherent light in scattering media, were developed $[18,19]$.

A plane ultrasonic wave is assumed to uniformly insonify a homogenous scattering medium. The optical mean free path is assumed to be much longer than the optical wavelength (weak scattering), and the acoustic amplitude is assumed to be much less than the optical wavelength (weak modulation). In this weak scattering approximation, the accumulative correlations between different random paths are negligible compared with the accumulative correlations from the photons traveling through the same pathlength $s$. The autocorrelation function of the scalar electric field, $E(t)$, of the scattered light can be expressed as

$$
G_{1}(\tau)=\int_{0}^{\infty} p(s)\left\langle E_{s}^{*}(t+\tau)\right\rangle d s .
$$

where the averaging is over time $t ; E_{s}$ is the unit electric field of the scattered light of path length $s$; and $p(s)$ is the probability density function of $s$. The contributions from Brownian motion and from the ultrasonic field to the autocorrelation function are independent; hence, they can be treated separately. For conciseness, only the ultrasonic contribution is considered here.

A coherent optical beam from an extended plane source is assumed to be incident upon a slab of thick- ness $L$, and the transmitted light is detected from a point. The function $p(s)$ is derived from diffusion theory with a zero-boundary condition [21]. Carrying out some lengthy algebra and calculus yields

$$
\begin{aligned}
& G_{1}(\tau)=\frac{(L / l) \sin h\left[\left\{\varepsilon\left[1-\cos \left(\omega_{a} \tau\right)\right]\right\}^{1 / 2}\right]}{\sin h\left[(L / l)\left\{\varepsilon\left[1-\cos \left(\omega_{a} \tau\right)\right]\right\}^{1 / 2}\right]} \\
& \left.\varepsilon=6\left(\delta_{n}+\delta_{d}\right) n_{0} k_{0} A\right)^{2}, \\
& \delta_{n}=\left(\alpha_{n 1}+\alpha_{n 2}\right) \eta^{2} \\
& \alpha_{n 1}=k_{a} l \tan ^{-1}\left(k_{a} l\right) / 2 \\
& \alpha_{n 2}=\alpha_{n 1} /\left[\left(k_{a} l\right) / \tan ^{-1}\left(k_{a} l\right)-1\right] \\
& \delta_{d}=1 / 6
\end{aligned}
$$

where $n_{0}$ is the background index of refraction, $k_{0}$ is the optical wave vector in vacuo, $A$ is the acoustic amplitude, $k_{a}$ is the acoustic wave vector; and $l$ is the transport mean free path. The parameter $\eta$ is related to the adiabatic piezo-optical coefficient of the material $\partial n / \partial p$, the density $\rho$, and the acoustic velocity $v_{a}$ : $\eta=(\partial n / \partial p) \rho v_{a}^{2}$.

The parameters $\delta_{n}$ and $\delta_{d}(=1 / 6)$ are related to the average contributions per free path and per scattering event, respectively, to the ultrasonic modulation of light intensity. The expression for $\delta_{n}$ is available analytically and is related to $k_{a} l$. The contribution from the index of refraction $\delta_{n}$ increases with $k_{a} l$ because a longer free path, relative to the acoustic wavelength, accumulates a greater phase modulation. By contrast, the contribution from displacement $\delta_{d}$ stays constant at $1 / 6$, independent of $k_{a}$ and $l$. As a result, the ratio between $\delta_{n}$ and $\delta_{d}$ increases with $k_{a} l$ and crosses a critical point at $k_{a} l=0.559$, where the ratio is unity. When $k_{a} l$ is less than this critical point, the contribution from the index of refraction is slightly less than the contribution from displacement; beyond this critical point, the contribution from the index of refraction increases and significantly can outmatch the contribution from displacement.

Based on the Wiener-Khinchin theorem, the power spectral density of the modulated speckles is related to the autocorrelation function $G_{1}(\tau)$ through the Fourier transformation:

$$
S(\omega)=\int_{-\infty}^{+\infty} G_{1}(\tau) \exp (i \omega \tau) d \tau
$$

The frequency $\omega$ is relative to the intrinsic optical frequency $\omega_{0}$ of the unmodulated laser beam because 


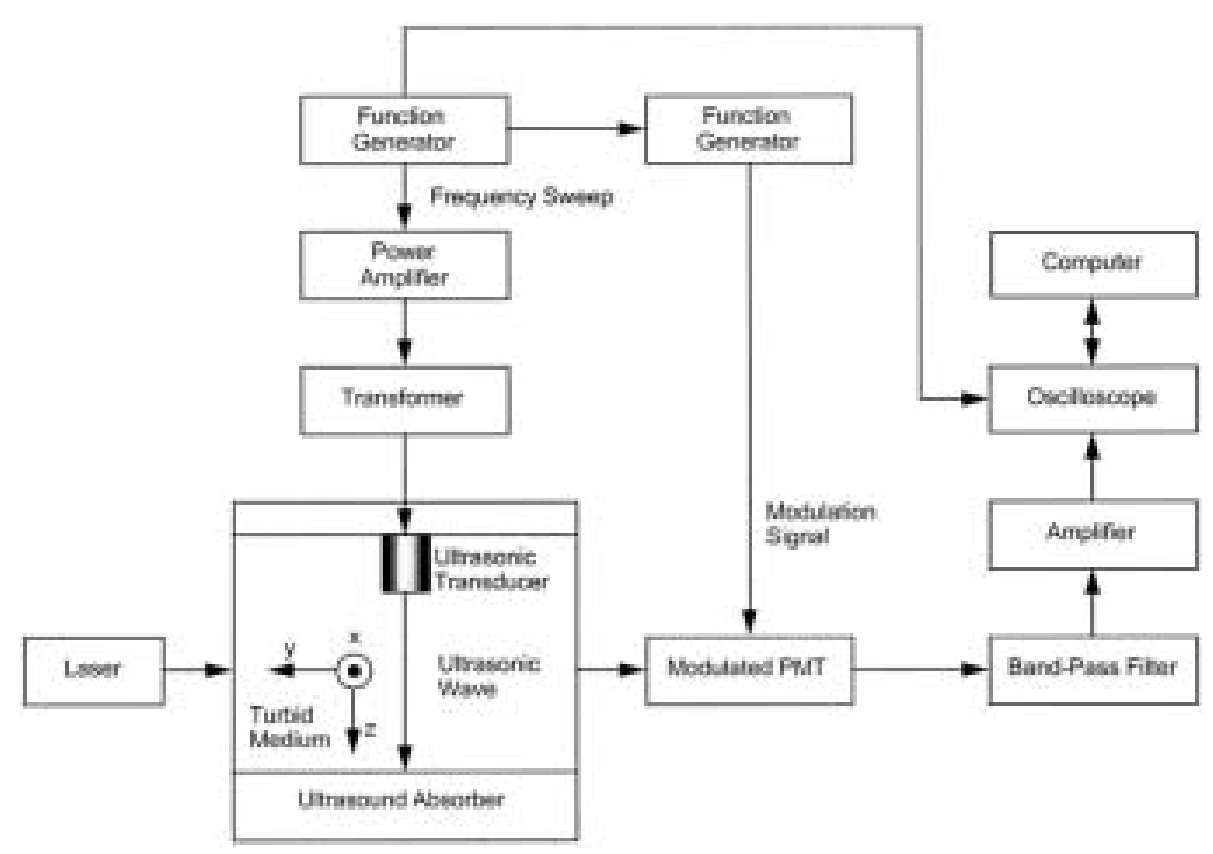

Fig. 1. Schematic of the experimental setup for Fourier-transform acousto-optical tomography (AOT).

the autocorrelation function $G_{1}(\tau)$ implicitly contains $\left.\exp )-i \omega_{0} \tau\right)$, which was dropped for convenience in the derivation. For example, $\omega=0$ in $S(\omega)$ corresponds to the intrinsic optical frequency $\omega_{0}$.

Since $G_{1}(\tau)$ is an even periodic function of $\tau$, the spectral intensity at frequency $n \omega_{a}$ can be calculated by

$$
I_{n}=\int_{0}^{T_{a}} \cos \left(n \omega_{a} \tau\right) G_{1}(\tau) d \tau / T_{a}
$$

where $T_{a}$ is the acoustic period. The frequency $n \omega_{a}$ is relative to the intrinsic optical frequency of the unmodulated laser beam $\omega_{0}$. The modulation depth $(M)$, defined as the ratio between the intensity at the fundamental frequency $I_{1}$ and the unmodulated intensity $I_{0}$, often is of experimental interest.

It is worth commenting that only the non-linear terms of phase accumulation contribute to the ultrasonic modulation of coherent light, as can be seen in Eq. 3 of Reference [18]. This is because the linear term vanishes as a result of optical random walk in scattering media. Under the condition of weak modulation, i.e., $(L / l) \varepsilon^{1 / 2}=\ll 1$, Eq. (2) can be simplified to $G_{1}(\tau)=1-(1 / 6)(L / l)^{2} \varepsilon\left[1-\cos \left(\omega_{a} \tau\right)\right]$. As a result, the modulation depth can be approximated to $M=(l / l)^{2} \varepsilon / 6 \propto A^{2}$-indicating a quadratic relationship between $M$ and the acoustic amplitude $A$, which was observed experimentally using a Fabry-Perot in- terferometer [5]. By contrast, acousto-optics in clear (non-scattering) media includes contributions from the linear term of phase accumulation. In this case, the modulation depth is linear with the acoustic amplitude $A$. Therefore, in the ballistic (non-scattering) regime, $M$ should be proportional to $A$; in the multiplescattering diffusion regime, $M$ should be proportional to the quadratic of $A$; in the quasi-ballistic (minimalscattering) regime, $M$ may show a mixed behavior with A.

If the modulated light is not detected with a spectrometer such as a Fabry-Perot interferometer, one needs to be cautious about the relationship between the detected apparent "modulation depth" $\left(M^{\prime}\right)$-defined as the ratio between the observed ac and dc signalsand the acoustic amplitude $A$. In many cases, the ac signal originates from the beat between the electric field at the fundamental frequency of the modulated light $\left(\omega_{0} \pm \omega_{a}\right)$ and the electric field at the intrinsic unmodulated optical frequency $\left(\omega_{0}\right)$. As a result, the apparent "modulation depth" will be approximately $M^{\prime} \propto\left(I_{1} / I_{0}\right)^{1 / 2}=M^{1 / 2} \propto A$, indicating that $\mathrm{M}^{\prime}$ is proportional to the acoustic amplitude $A$.

\section{Acousto-optical tomography: Experiment}

In this section, the first two subsections review Fourier-transform AOT [11], the second two subsec- 
tions review AOT based on parallel detection [12], and the last subsection demonstrates the virtual optical source defined by the ultrasound [22], which is the basis of AOT.

\subsection{Fourier-transform acousto-optical tomography: Experimental setup}

In this technique, a frequency-swept (chirped) ultrasonic wave was used to encode laser light that crosses the acoustic axis of the ultrasonic transducer with various frequencies. Decoding the transmitted light in the frequency domain allowed us to image, with resolution along the acoustic axis, objects buried inside the scattering media. This encoding scheme is analogous to that of magnetic resonance imaging (MRI).

A block diagram of the experimental setup is shown in Fig. 1. A frequency-swept signal was produced using a function generator (Stanford Research System, DS345) and then was amplified in power and amplitude by a power amplifier (ENI, 325LA-HP) and a transformer, respectively. The instantaneous frequency of the frequency sweep was

$$
f_{s}(t)=a_{s}+b t
$$

where as was the starting frequency, $b$ was the sweep rate, and $t$ was the time. In our experiment, the frequency sweep had a $3-\mathrm{MHz}$ bandwidth between 7.0 MHz and $10.0 \mathrm{MHz}$, with a sweep rate of $297 \mathrm{MHz} / \mathrm{s}$. The amplified signal was applied to an ultrasonic transducer (Panametrics, V312-SU). The ultrasonic wave was propagated vertically into a scattering medium, which was contained in a glass tank. An ultrasound absorber was placed at the bottom of the tank to avoid ultrasonic reflection from the water-glass interface.

A He-Ne laser (Uniphase, 1145P) beam at the 633$\mathrm{nm}$ wavelength, after being broadened to $15 \mathrm{~mm}$, illuminated the scattering medium perpendicularly to the ultrasonic column. The ultrasonic column modulated the laser light. The instantaneous frequency of ultrasonic modulation along the column was

$$
f_{s}(t, z)=a_{s}+b\left(\left[t-\frac{z-z_{0}}{v_{s}}\right]\right),
$$

where $z$ was the ultrasonic axis, $z_{0}$ was the point of ultrasonic emission in the ultrasonic transducer, and vs was the speed of sound in the medium. The above equation was valid for $t \leqslant\left(z-z_{0}\right) / v_{s}$. A photomultiplier tube (PMT) (Hamamatsu, R928) collected some transmitted light and converted the optical power into (a)

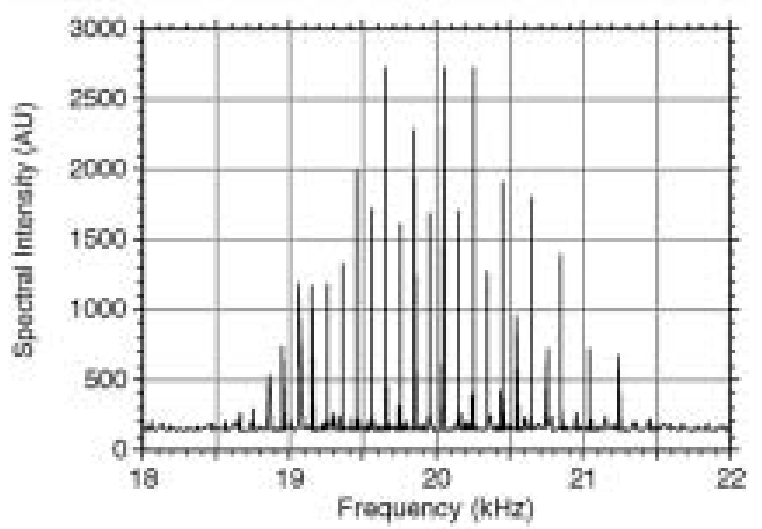

(b)

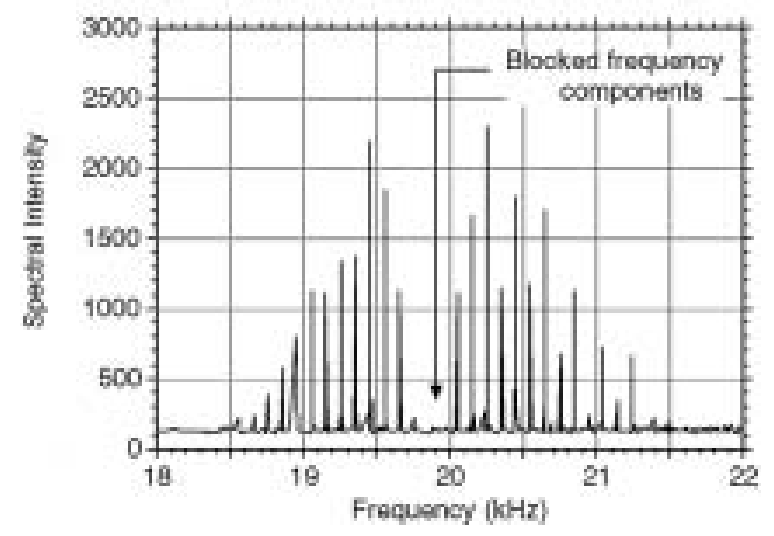

Fig. 2. a. Frequency spectrum of the heterodyned frequency-swept ultrasound-modulated optical signal when the object was far from the ultrasonic axis. b. Frequency spectrum when the object was on the ultrasonic axis.

an electric signal. The gain of the PMT was modulated for heterodyne detection by a reference signal produced from another function generator (Stanford Research System, DS345). The reference modulation signal also was frequency-swept and had an instantaneous frequency of

$$
f_{r}(t)=a_{r}+b t
$$

where the starting frequency $a_{r}$ of the reference modulation signal was different from that of the signal for the ultrasonic transducer.

The frequency of the heterodyned signal contributed from the ultrasonic modulation at $z$ was

$$
\begin{aligned}
f_{h}(t, z) & =\left|f_{s}(t, z)-f_{r}(t)\right| \\
& =\left|a_{s}-a_{r}-\frac{b\left(z-z_{0}\right)}{v_{s}}\right|,
\end{aligned}
$$

which was independent of time $t$. The electronically heterodyned signal at the output of the PMT was band- 

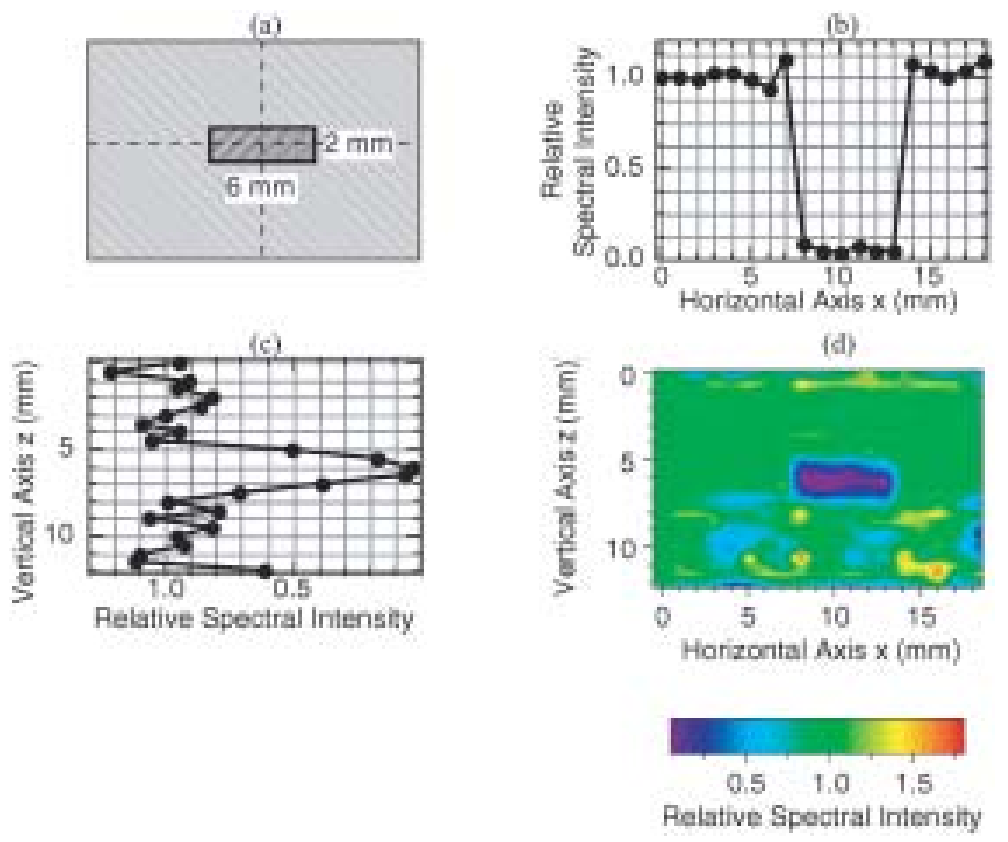

Fig. 3. a. Cross-sectional view of the object buried in a scattering medium. b. One-dimensional (1D) image of the scattering medium horizontally across the center of the object as indicated by the horizontal dashed line in panel a. c. 1D image of the scattering medium vertically across the center of the object as indicated by the vertical dashed line in panel a. d. Two-dimensional tomographic image of the object buried in a scattering medium.

pass filtered and then was amplified using a filter and an amplifier (Stanford Research System, SR650), respectively. The bandwidth of the filter was determined by a desired range of $z$ to be imaged (zone of detection) as follows:

$$
\Delta f_{h}=\frac{b}{v_{s}} \Delta z
$$

where $\Delta z$ was the range of $z$ to be detected. The signal from the amplifier was recorded by a digital oscilloscope (Tektronix, TDS540B) and then transferred to a computer for post-processing.

An object made of rubber was buried in the middle plane of the tank. The thickness of the tank along the laser beam was $17 \mathrm{~cm}$. The object was translated in the tank along the $\mathrm{x}$-axis perpendicularly to both the laser beam and the ultrasonic column. A time-domain signal was recorded at each stop. A fast Fourier transform (FFT) was used to compute the spectra of the recorded time-domain signals on a computer.

The frequency spectra yielded imaging information for the zone of detection. The frequency in the spectra corresponded to the difference between the instantaneous ultrasonic frequency at an ultrasonic axial position and the instantaneous frequency of the reference modulation signal. By using Eq. (13), the frequency spectra were converted into 1D images of the scattering medium along the ultrasonic axis ( $\mathrm{z}$ axis). The imaging contrast corresponded to the spatial variation in the optical and acoustic properties.

\subsection{Fourier-transform acousto-optical tomography: Experimental results}

Two sample frequency spectra are depicted in Fig. 2. Figure 2(a) shows the spectrum when the object was far from the ultrasonic axis, and Fig. 2(b) shows the spectrum when the object blocked part of the laser beam along the ultrasonic axis. Some of the frequency components, corresponding to the location of the object, disappeared as indicated in Fig. 2(b). This figure demonstrates the one-to-one correspondence between heterodyned frequency and position along the ultrasonic axis.

Multiple 1D spectra were acquired while the buried object was scanned horizontally along the x-axis with a step size of $1 \mathrm{~mm}$. The first spectrum, which was taken when the object was far from the ultrasonic axis, was used as a reference spectrum. An alternative would be to use the average of all of the acquired spectra as the reference. The spectral intensities of the subsequent spectra were divided by that of the reference spectrum to obtain a relative spectral intensity. A 2D image of 
the medium was formed by combining the $1 \mathrm{D}$ relative spectra at the various $x$ positions.

A 2D image of the object buried in a scattering medium is shown in Fig. 3. The corresponding 1D images in both the $x$ and the $z$ directions were examined in detail as shown in Fig. 3(b) and Fig. 3(c), respectively. As can be seen, the edge resolution in both the $\mathrm{x}$ - and z-axes was approximately $500 \mu \mathrm{m}$ (50\% drop) with the $3-\mathrm{MHz}$ bandwidth ultrasonic sweep. The z-axis resolution was limited by the ultrasonic sweep parameters, and the $\mathrm{x}$-axis resolution was limited by the ultrasonic focus.

\subsection{Parallel acousto-optical tomography: Experimental setup}

The experimental setup is shown in Fig. 4. A coordinate system was established for reference. The $\mathrm{z}-$ axis was on the acoustic axis pointing from the ultrasonic transducer to the sample. The y-axis was along the optical axis pointing to the diode laser. The $\mathrm{x}$-axis was perpendicular to both the acoustic and the optical axes. Ultrasonic waves were generated by a focused ultrasonic transducer (Panametrics, V314) with a $2.54-\mathrm{cm}$ focal length in water and a $1-\mathrm{MHz}$ central response frequency. The peak pressure at the focus was $\sim 2 \times 10^{5} \mathrm{~Pa}$, well below the damage threshold for biological tissue [23]. The laser beam from a diode laser (Melles Griot, 56IMS667, 690-nm wavelength) was expanded to $1.6 \mathrm{~cm} \times 0.3 \mathrm{~cm}$ and projected onto the tissue sample. The average power and the coherence length of the modulated laser beam were $12 \mathrm{~mW}$ and $\sim 7 \mathrm{~cm}$, respectively. The tissue sample was partially immersed in water for good acoustic coupling. The light transmitted through the tissue sample generated a speckle pattern, which was detected by a high-speed 12-bit digital CCD camera (Dalsa CA-D10256T). Three function generators (Stanford Research Systems, DS345), FG-1, FG-2, and FG-3, shared the same time base to ensure synchronization. FG-1 and FG-2 generated chirp functions to modulate the diode laser and to excite the ultrasonic transducer, respectively. A delay generator, DG, controlled the time delay between the trigger signals to FG-1 and FG-2.

If the signal from FG-2 had not been amplitudemodulated by FG-3, the frequency of the heterodyne signal from location $z$ along the ultrasonic axis ( $z$ axis) would have been

$$
f_{h}(z, \tau)=b\left(\tau-\frac{z}{v_{s}}\right)
$$

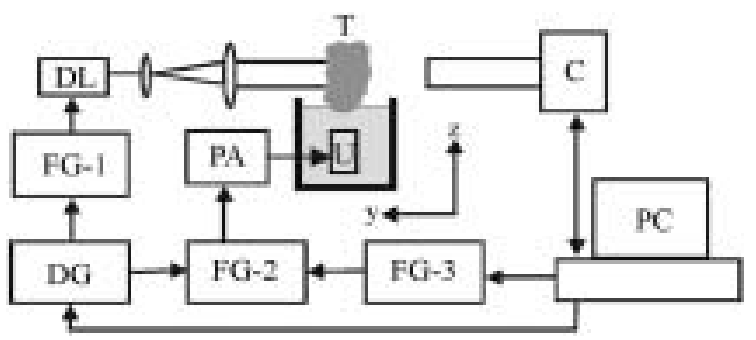

Fig. 4. Schematic of the experimental setup for parallel acousto-optical tomography (AOT). DL: diode laser; C: CCD camera; U: ultrasonic transducer; FG-1, FG-2 and FG-3: function generators; DG: delay generator; PA: power amplifier; T: tissue sample.

where $b$ is the rate of the frequency sweep, $v_{s}$ is the ultrasonic velocity, and $\tau$ is the time delay between the two chirps from FG-2 and FG-1. To implement the source-synchronized lock-in technique, we used FG-3 to produce a reference sinusoidal wave with a frequency equal to $f(z d \tau)$, which modulated the amplitude of the chirp function generated by FG-2. The signal in a single CCD pixel can be represented as

$$
I_{i}\left(\phi_{i}\right) \propto I_{b}+I_{m} \cos \left(\phi_{s}+\phi_{r}\right),
$$

where $I_{b}$ is the background intensity, $I_{m}$ is the signal intensity related to the ultrasound-modulated component, $\phi_{s}$ is a randomly distributed initial phase of the speckle that does not provide useful information in our imaging system, and $\phi_{r}$ is the initial phase of the reference sinusoidal wave from FG-3. The quantity $M^{\prime}=I_{m} / I_{b}$ is related to the modulation depth, which reflects the local optical and ultrasonic properties. The initial phase $\phi_{r}$ of this sinusoidal wave was set sequentially to $0^{\circ}, 90^{\circ}, 180^{\circ}$, and $270^{\circ}$. The corresponding four frames of CCD images were acquired to calculate M' as follows

$$
\begin{aligned}
& M^{\prime}=\frac{1}{2 I_{b}} \\
& \sqrt{\left(I_{i}\left(90^{\circ}\right)-I_{i}\left(270^{\circ}\right)\right)^{2}+\left(I_{i}\left(0^{\circ}\right)-I_{i}\left(180^{\circ}\right)\right)^{2}} .
\end{aligned}
$$

The above calculation was performed for each pixel of the CCD camera to recover $M$; this is an extremely fast procedure with today's computers. A total of $256 \times$ 256-pixel data points were then averaged to produce a single data point for the image.

The reference (lock-in) frequency from FG-3 was set to a fixed value $f_{r}$. The ultrasound-modulated light from a specific spatial location $z_{0}$ corresponding to the heterodyne frequency $f_{r}$ and the time delay $\tau$ was detected, where $z_{0}$ can be derived from Eq. (15):

$$
z_{0}=v_{s}\left(\tau-\frac{f_{r}}{b}\right) \text {. }
$$



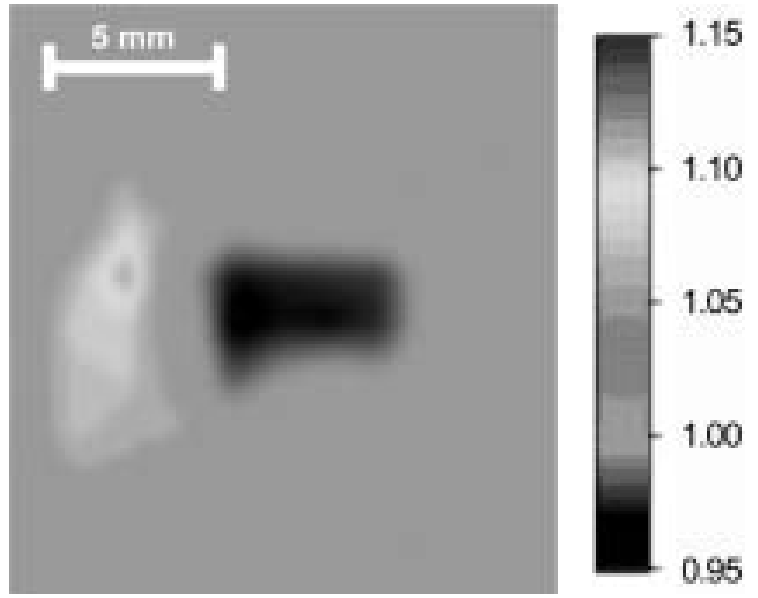

Fig. 5. Experimental two-dimensional image of 1.2-cm thick chicken breast tissue containing a buried object. The horizontal and vertical axes are along the $x$ and $z$ axes, respectively.

The ultrasound-modulated light from the other spatial locations had different frequencies and hence was rejected by the CCD camera. We obtained 1D images along the ultrasonic axis by electronically scanning the time delay $\tau$. To obtain 2D tomographic images, we mechanically scanned the ultrasonic transducer with a translation stage along the $\mathrm{x}$-axis.

\subsection{Parallel acousto-optical tomography: Experimental results}

Skinless chicken breast tissues were used as samples in the experiments. The objects buried in the tissue were made from soft rubber, which has good acoustic coupling with tissue and has little acoustic absorption. Figure 5 shows a 2D image obtained from a $1.2-\mathrm{cm}$ thick chicken breast tissue sample with an object buried inside. The size of the object was $4.0 \mathrm{~mm} \times 6.2 \mathrm{~mm}$ $\times 2.7 \mathrm{~mm}$ along the $x, y$, and $z$ axes, respectively. The step size of the electronic scan along the $z$-axis was $1.0 \mu \mathrm{s}$, corresponding to $\sim 1.5 \mathrm{~mm}$ in the tissue. The step size of the mechanical scan along the $x$-axis was $1.5 \mathrm{~mm}$. The buried object is clearly visible in the background. The image resolution along the $x$-axis is $\sim 2 \mathrm{~mm}$, which is determined by the $2-\mathrm{mm}$ focal diameter of the ultrasonic transducer.

The spatial resolution along the ultrasonic axis $(z$ axis) $\Delta z$ is determined by the frequency span $\Delta f$ of the chirp function and the ultrasonic velocity $v_{s}$ as follows,

$$
\Delta z \approx v_{s} / \Delta f
$$

where $v_{s} \approx 1,500 \mathrm{~m} / \mathrm{s}$. The spatial resolution can be scaled along the ultrasonic axis by changing the frequency span $\Delta f$. To demonstrate this scalability, we acquired two $1 \mathrm{D}$ images with frequency spans $\Delta f=$ $800 \mathrm{kHz}$ and $\Delta f=400 \mathrm{kHz}$ and observed that the corresponding spatial resolutions were 2 and $4 \mathrm{~mm}$, respectively.

To rule out the possibility of the collection of ballistic photons, we acquired a $1 \mathrm{D}$ ultrasound-modulated optical image with the laser beam illuminating the sample obliquely at $10^{\circ}$ to the $z$-axis. Any ballistic photons, if they existed, would have missed the detector. The imaging signal $\left(M^{\prime}\right)$ and the image measured in the case of oblique incidence were almost the same as those measured in the case of normal incidence. From this result, it is clear that ballistic photons are not the major contributors to the signal in our experiments and that AOT depends primarily on scattered photons.

\subsection{Demonstration of the virtual optical source defined by the ultrasound}

As shown in Eq. (15), the frequency of the heterodyned signal is related to the source location $y$. Consider the ultrasound-modulated optical signal at $y$ of the ultrasonic column as a virtual source. When the virtual source propagates through a scattering medium, a direct view of the virtual source is blurred. However, if the virtual source is detected immediately without further propagation, a clear view of the virtual source can be acquired. Figure 6 shows a series of images corresponding to various values of y obtained by adjusting the frequency $f_{h}$ [22]. This demonstration clearly illustrates the importance of ultrasound tagging of light that enhances the spatial resolution of imaging. A small light source that is scanned inside the highly scattering medium can produce a better image of the scanned cross-section than can a small light source that is scanned outside the medium.

\section{Photo-acoustic tomography: Introduction}

Photo-acoustic tomography (PAT) in biological tissues, also called opto-acoustic or thermo-acoustic tomography, is another ultrasound-mediated biophotonic imaging modality. In PAT, a short-pulsed electromagnetic source-a laser, for example-is used to irradiate the tissue samples. Although a general consensus has not been reached, if the heating source is a laser, the technology often is referred to as photo-acoustic or optoacoustic tomography; if the heating source is in the radiofrequency band, the technology usually is referred 

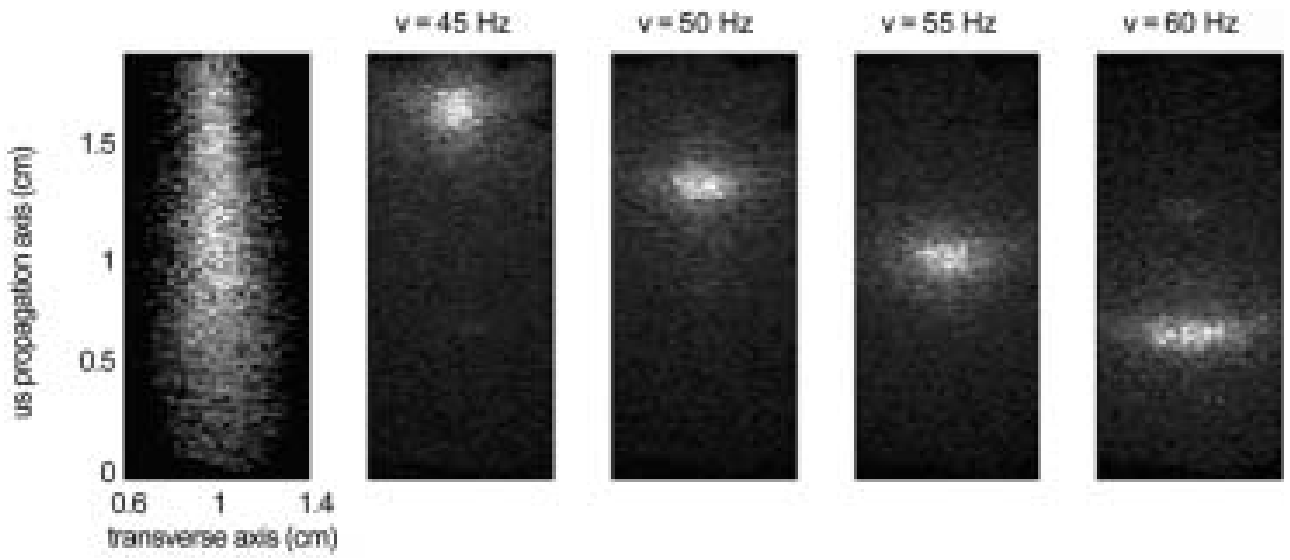

Fig. 6. Demonstration of the virtual source of ultrasound-modulated light. Left frame: the entire virtual source. Following frames: virtual sources corresponding to various values of y obtained by adjusting the frequency fh.

to as thermo-acoustic tomography. The photo-acoustic waves excited by thermoelastic expansion are measured around the sample by wideband ultrasonic transducers, which are sensitive to small vibrations. The electromagnetic heating must be rapid to produce photoacoustic waves efficiently; in other words, static temperature distribution or slow heating does not produce photo-acoustic waves effectively. The acquired photoacoustic waves then are used to deduce the electromagnetic absorption distribution. Regardless of whether a laser source or a radiofrequency source is used, the data collection and image reconstruction are conducted in the same way. The sections that follow are devoted to the reconstruction theory-which is common to both the laser- and radiofrequency-based approaches-and to laser-based PAT.

Although various groups have studied PAT, only representative works are summarized briefly here. The sensitivity of PAT to detect small embedded tumors has been explored [24] as well as its ability to detect objects in layered tissues such as the skin [25]. It also has been shown that PAT can image blood vessels with high resolution [26], and an optical method has been demonstrated for 2D ultrasonic detection [27,28]. In addition, sector-scan PAT with a focused ultrasonic transducer has been developed [29], and reconstruction-based PAT has been studied [30-31323334].

The key task in PAT is to determine the electromagnetic absorption distribution from the measured photoacoustic data; i.e., to map the electromagnetic absorption heterogeneity of the tissue. One straightforward approach is to use focused ultrasonic transducers to localize the photo-acoustic sources in linear or sector scans and then construct the images directly from the data $[29,35,36]$, as was done in early pulse-echo ultrasonography. An alternative method is to use wideband unfocused detectors to acquire photo-acoustic data and then reconstruct the electromagnetic absorption distribution. The reconstruction is an inverse source problem similar to that in positron emission tomography (PET); however, the reconstruction in PET is based on geometric optics, whereas the reconstruction in PAT is based on diffractive (wave) optics. Reconstruction algorithms include the weighted delay-and-sum method [37], the optimal statistical approach [38], the Radon transform in far-field aption [39], and the back-projection method based on the Fourier transform [31-34].

\section{Photo-acoustic tomography: Reconstruction}

\subsection{Fundamentals of photo-acoustics: The forward problem}

Photo-acoustic theory has been discussed in many literature reviews such as in reference [40]. Here, we briefly review only the fundamental equations. If the electromagnetic pumping pulse duration is much shorter than the thermal diffusion time, thermal diffusion can be neglected; this is known as the assumption of thermal confinement. In this case, the acoustic wave $p(\mathbf{r}, \bar{t})$ is related to electromagnetic absorption, $H(\mathbf{r}, \bar{t})$, by the following wave equation [41]:

$$
\frac{\partial^{2} p(\mathbf{r} \bar{t})}{\partial \bar{t}^{2}}-\nabla^{2} p(\mathbf{r}, \bar{t})=\frac{\beta v_{s}}{C} \frac{\partial H(\mathbf{r} \bar{t})}{\partial \bar{t}},
$$

where $\bar{t}=t v_{s} ; v_{s}$ is the acoustic speed, assumed to be constant; $C$ is the specific heat; and $\beta$ is the coefficient 


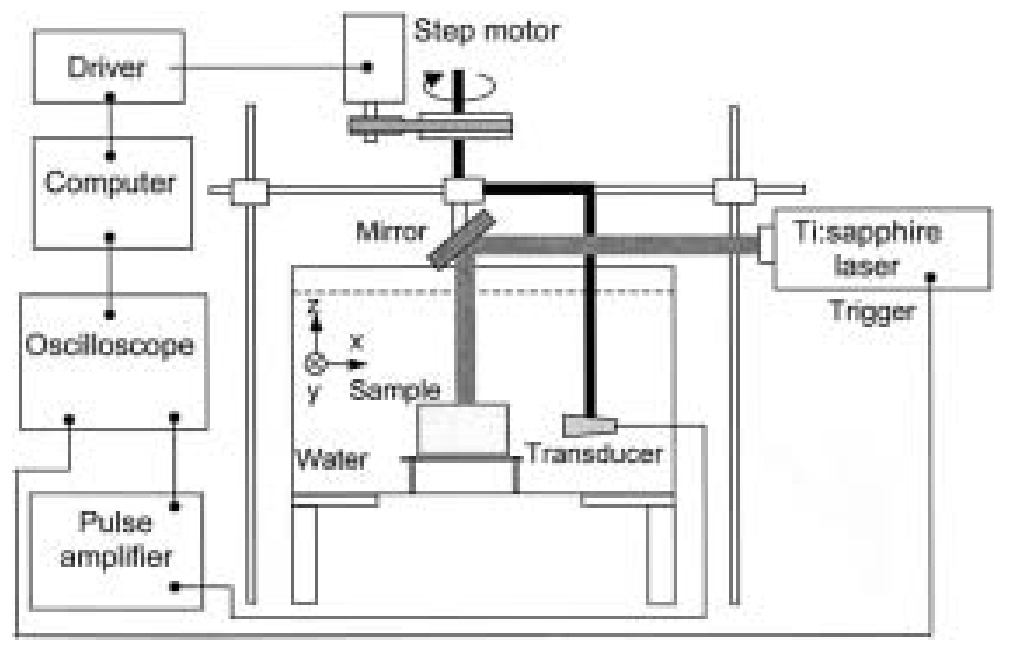

Fig. 7. Schematic of the experimental setup for photo-acoustic tomography (PAT).

of volume thermal expansion. Equation (20) can be rewritten in terms of $H(\mathbf{r}, \bar{t})$ :

$$
p(\mathbf{r}, \bar{t})=\frac{\beta v_{s}}{4 \pi C} \iiint \frac{\partial H\left(\mathbf{r}^{\prime} t^{\prime}\right)}{\partial t^{\prime}} \frac{d \mathbf{r}^{\prime}}{\left|\mathbf{r}-\mathbf{r}^{\prime}\right|},
$$

where $t^{\prime}=\bar{t}-\left|\mathbf{r}-\mathbf{r}^{\prime}\right|$. The source term $H(\mathbf{r}, \bar{t})$ can further be written as the product of a purely spatial and a purely temporal component, i.e.:

$$
H(\mathbf{r}, \bar{t})=I_{0} \varphi(\mathbf{r}) \eta(\bar{t}),
$$

where $\mathbf{I}_{0}$ is a scaling factor proportional to the incident radiation intensity; $\varphi(\mathbf{r})$ describes the electromagnetic absorption properties of the medium at $\mathbf{r}$; and $\tau(\bar{t})$ describes the shape of the irradiating pulse. Substituting Eq. (22) into Eq. (21) results in

$$
p(\mathbf{r}, \bar{t})=\frac{I_{0} \beta v_{s}}{4 \pi C} \iiint \varphi\left(\mathbf{r}^{\prime}\right) \frac{d \eta\left(t^{\prime}\right)}{d t^{\prime}} \frac{d \mathbf{r}^{\prime}}{\left|\mathbf{r}-\mathbf{r}^{\prime}\right|}
$$

This equation shows the solution to the forward problem-prediction of the pressure outside the tissue if the absorption properties of the medium and the profile of the electromagnetic pulse are known. In reality, the inverse problem needs to be solved for imaging.

\subsection{Modified back-projection: The inverse problem}

Exact inverse solutions in planar, spherical, and cylindrical geometries can be found in References [3134]. These exact solutions are computationally intensive and can be approximated to more efficient solutions in most cases $[42,43]$. In practice, the distance between the photo-acoustic sources and the detector is much longer than the wavelengths of the high-frequency photo-acoustic waves that are useful for imaging. Under this condition, the following back-projection algorithm is a good approximate inverse solution:

$$
\begin{gathered}
\varphi(\mathbf{r})=C \int_{S_{0}} \int d S_{0} \cos \left(\theta_{d}\right) \frac{1}{t} \\
\left.\frac{\partial p\left(\mathbf{r}_{0}, t\right)}{\partial t}\right|_{t=\left|\mathbf{r}_{0}-\mathbf{r}\right| / V_{s},}
\end{gathered}
$$

where $C$ is a constant, $S_{0}$ is the surface of detection, and $\theta_{d}$ is the angle between the normal of $d S_{0}$ and $\mathbf{r}-\mathbf{r}_{0}$ (the vector pointing from a point of detection to a point of reconstruction). It should be noted that this is a modified back-projection of quantity $\frac{1}{t} \frac{\partial p\left(\mathbf{r}_{0}, t\right)}{\partial t}$.

This back-projection is analogous to that in $\mathrm{x}$-ray computed tomography (CT). In x-ray CT, the backprojection is along the paths of $\mathrm{X}$-ray propagation. In PAT, the back-projection is along spherical shells that are centered at the detector and have a radius determined by the acoustic time of flight.

\section{Photo-acoustic tomography: Experiment}

\subsection{Experimental setup}

The schematic of a sample experimental setup for PAT [44] is shown in Fig. 7, where a laboratory coordinate system $(x, y, z)$ also is depicted. A Ti: sapphire laser was used as the light source and operated at a wavelength of $789.2 \mathrm{~nm}$, a pulse energy of $\sim 30 \mathrm{~mJ}$, a pulse duration of $4.7 \mathrm{~ns}$ FWHM, and a repetition rate of $10 \mathrm{~Hz}$. The laser beam was expanded to $1.5 \mathrm{~cm}$ in diameter and provided an incident power density within the ANSI standard for human skin [45]. A wideband unfocused ultrasonic transducer (V323, Panametrics) with 


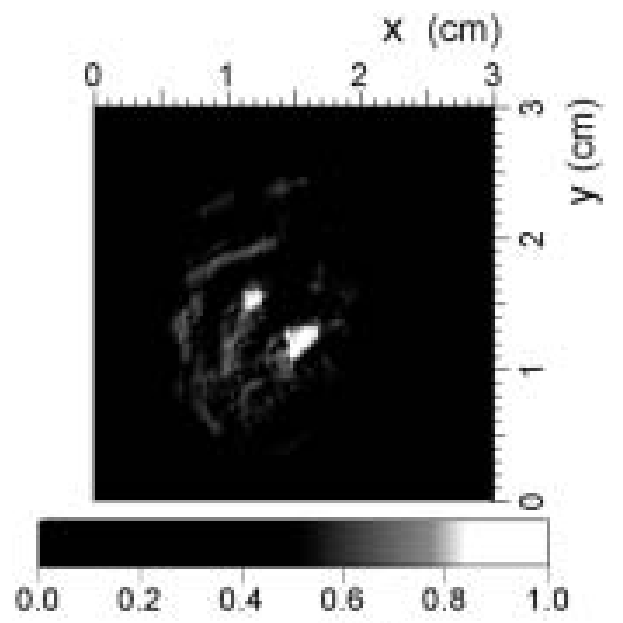

(a)

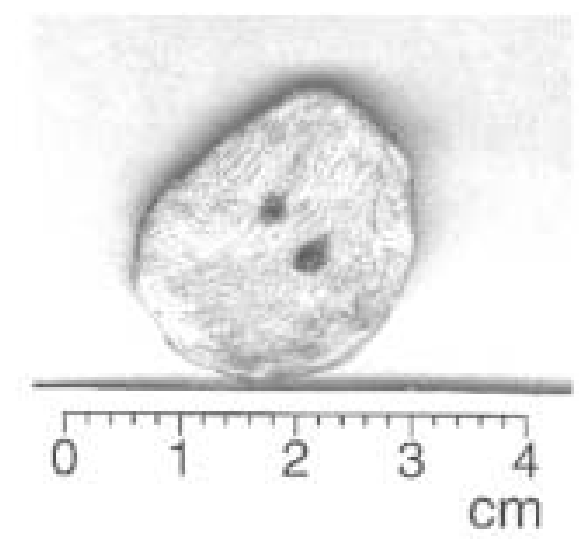

(b)

Fig. 8. Photo-acoustic tomography (PAT) of two slices of chicken gizzard embedded $0.5 \mathrm{~cm}$ deep in a chicken breast slab. a. Reconstructed image. b. Photography of the imaged cross-section of the sample.

a $2.25-\mathrm{MHz}$ central frequency and a $6-\mathrm{mm}$ diameter active element was used to receive the photo-acoustic waves. The transducer was scanned around the sample with a step size of $1.1^{\circ}$ and a radius of $5 \mathrm{~cm}$. The ultrasonic transducer and the sample were immersed in water for acoustic coupling. A low-noise pulse amplifier (500 PR, Panametrics) amplified the signals from the ultrasonic transducer. The amplified signals were collected by an oscilloscope. The digitized signals were averaged 30 times and then transferred to a computer for image reconstruction.

\subsection{Photo-acoustic images of phantoms}

Using the above experimental setup, thin slices of gizzard tissues placed $0.5 \mathrm{~cm}$ deep in chicken breast muscle slabs were imaged. The blood concentration in the chicken gizzard tissue is much higher than that in the chicken breast muscle. Therefore, the gizzard is more strongly absorbing than is the breast tissue. Image reconstruction utilized the $2 \mathrm{D}$ modified backprojection algorithm [32], in which $1.5 \mathrm{~mm} / \mu$ s was used as the sound velocity in the soft tissues. The reconstructed image and a photograph of the sample demonstrate good agreement between them (Fig. 8). It was shown experimentally that the spatial resolution is diffraction-limited by the photo-acoustic waves [32].

\subsection{Photo-acoustic images in situ and in vivo}

PAT also has been used to image small animals and humans. Examples are provided in this subsection to illustrate the potential applications of PAT. Using the 532-nm wavelength, blood vessels in the cortical surface of small animals can be imaged transcranially with the skin and the skull intact (Fig. 9) [46]. The contrast is quite high at this wavelength; however, the imaging depth is limited to $1 \mathrm{~cm}$ or so. Nevertheless, this depth is enough for the imaging of the entire brain of a small animal [47].

Using the 1064-nm wavelength, the imaging depth is expected to be enhanced at the expense of reduced contrast. Nonetheless, there still is enough contrast for breast cancer detection, even at this wavelength (Fig. 10) [48], which demonstrates the advantages of optical contrast. The patient had a ductal carcinoma measuring $6 \times 7 \mathrm{~mm}^{2}$ with its core $11 \mathrm{~mm}$ beneath the breast surface, as shown by the ultrasound image. The long curved features that extend downward from the tumor are artifacts that result from the limited view of detection due to the small number of detectors in this current imaging system [49]. These artifacts can be eliminated if a full-view detector array is put into operation.

\section{Summary and discussion}

Neither AOT nor PAT depend on ballistic/quasiballistic or backscattered light as does OCT. Any light, including both singly and multiply scattered photons, contributes to the imaging signals. As a result, the imaging depth in AOT or PAT is extended compared with other ballistic/quasi-ballistic imaging modalities. Furthermore, both AOT and PAT are free of the speckle artifacts present in OCT and pulse-echo ultrasonography, two analogous technologies. The advantages of AOT and PAT are summarized as follows: 


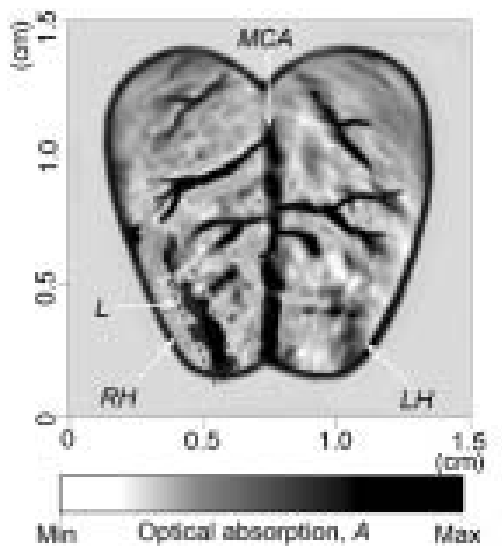

(a)

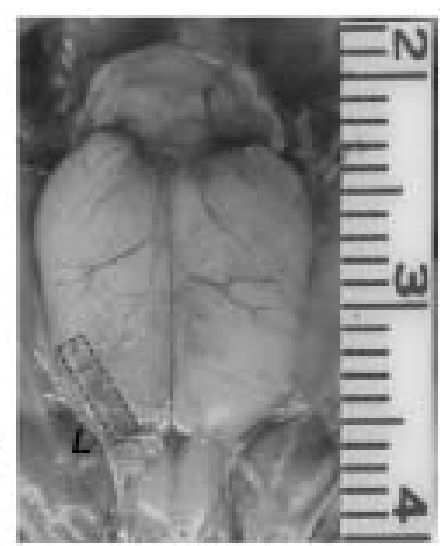

(b)

Fig. 9. a. Non-invasive PAT image of a superficial lesion $(1 \mathrm{~mm} \times 4 \mathrm{~mm}$, in the right cortex cerebri-area frontalis $)$ on a rat's cerebra acquired with the skin and skull intact. RH: right cerebral hemisphere; LH: left cerebral hemisphere; and L: lesion. The blood vessels distributed on both sides of the middle cerebral artery (MCA) are imaged clearly. The matrix size of the image was 1000 (horizontal) $\times 1000$ (vertical), showing a $1.5 \mathrm{~cm} \times 1.5 \mathrm{~cm}$ region. b. Open-skull photograph of the rat cerebral surface acquired after the PAT experiment.

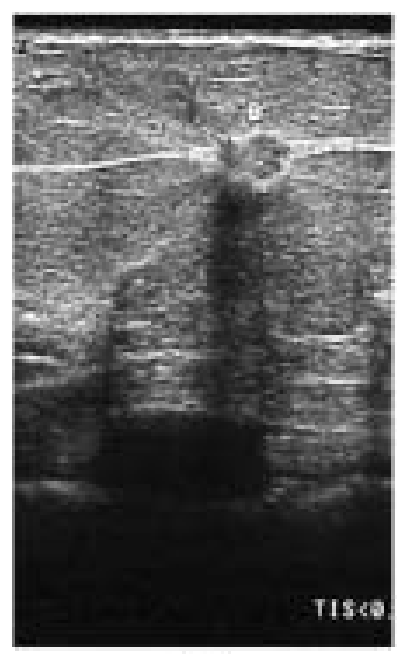

(a)

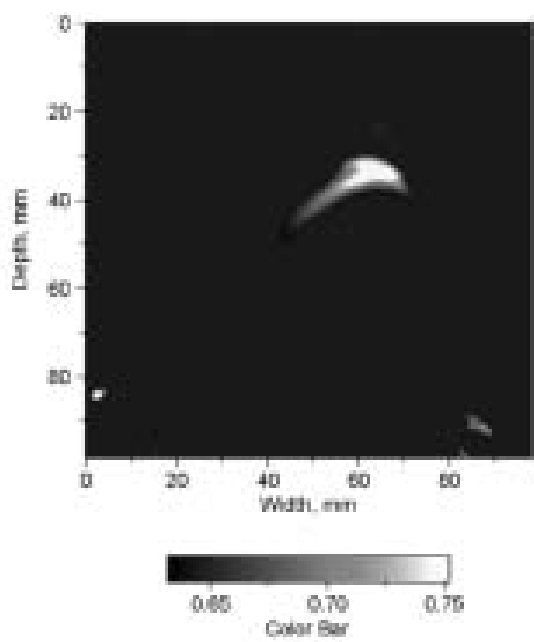

(b)

Fig. 10. a. Conventional ultrasound B-scan image of a human breast containing a tumor (ductal carcinoma). b. Non-invasive PAT image of the same breast in vivo. (Reprinted from mA.A. Oraevsky, A.A. Karabutov, V.S. Solomatin, E.V. Savateeva, V.G. Andreev, Z. Gatalica, H. Singh and R.Y.D. fleming, Laser optoaccoustic imaging of breast from the Society of Photo-Optical Instrunmentation Engineers.)

- Combination of high electromagnetic contrast and high acoustic resolution.

- Potential for simultaneous functional imaging of blood oxygenation and blood volume. In comparison, functional magnetic resonance imaging (fMRI) directly measures only the deoxygenated hemoglobin.

- High ratio between imaging depth and resolution.

- No speckle artifacts.
- Scalable resolution and imaging depth by varying the ultrasonic frequency.

- Ability to simultaneously acquire AOT/PAT images and pure ultrasound images from the same cross-sections of the sample for added diagnostic value. The two types of images can be coregistered naturally.

- Use of non-ionizing radiation that poses no known hazards to humans. Both the laser and ultrasound in AOT or PAT are non-ionizing radiation and ir- 
radiate biological tissue within the safety limits.

Because of the use of ultrasound, AOT and PAT inherit some of the limitations that are associated with the ultrasound technology. Contact measurements are required for acoustic coupling in AOT and PAT. The human skull, for instance, poses strong ultrasonic wavefront aberrations, although corrections theoretically are possible. Heretofore, acoustic heterogeneity has not been taken into account experimentally in AOT and PAT [50]. In cases when acoustic heterogeneity is strong, concurrent ultrasound imaging should be useful for providing the acoustic properties that are needed for image reconstructions in AOT or PAT.

\section{Outlook}

Although they undoubtedly are in their infancy, both AOT and PAT are emerging technologies that combine good acoustic resolution with strong optical contrast and sensitivity. The full mechanisms of AOT were understood only recently [18], although the mechanisms for PAT have been known for over a century. The theoretical understanding of AOT is expected to advance its experimental realization. Because the imaging depth and resolution are scalable with the acoustic frequency, the potential for applications in biological tissues that are accessible by the adjustable imaging depth are broad. AOT can image up to $5 \mathrm{~cm}$ deep into chicken tissue with a $2-\mathrm{mm}$ resolution at a $1-\mathrm{MHz}$ ultrasonic frequency. Laser-based PAT has been shown to image up to $2-3 \mathrm{~cm}$ into chicken tissue with good resolution and can image even deeper at the cost of resolution. Radiofrequency-based PAT can image multiple centimeters into soft tissue due to the good penetration of radiofrequency waves; therefore, it is believed to be a strong contender for breast imaging. Both AOT and PAT can be scaled down to provide microscopic imaging using high-frequency ultrasound. The applications can target superficial lesions in, for example, the skin, the oral cavity, and the cervix. Multiple optical wavelengths can be used to extract physiological parameters such as oxygen saturation of hemoglobin and blood concentration. Ultrasound arrays will improve the speed and accuracy of data acquisition significantly. When they are combined with molecular contrast agents, AOT and PAT also are enabling technologies for molecular imaging, and they will provide better spatial resolution than do all-optically based approaches. In closing, although the development of AOT and PAT has reached an exciting phase, it is clear that their full potential in medical science have yet to be achieved.

Other ultrasound-mediated optical imaging modalities deserve mention here. Ultrasonograms have been used to provide a priori structural information for the quantitative characterization of functional optical signatures of embedded tumors [51]. This combination overcomes the instability in pure optical reconstruction. Sonoluminescent tomography, based on the generation of light with ultrasound, also has been explored to image scattering media [52]; it may find applications in monitoring ultrasound therapeutics with optical information.

\section{Acknowledgements}

The author acknowledges the contributions from his many talented group members and collaborators as indicated by the cited references. The author also is grateful to the organizers of this special issue at the National Institutes of Health (NIH) for inviting his participation. The research reported here has been sponsored in part by NIH Grant No. R29 CA68562, No. R01 CA71980, No. R21 CA83760, and No. R01 EB000712; the National Science Foundation Grant No. BES-9734491; the US. Army Medical Research and Material Command Grant No. DAMD 17-00-1-0455; and the Texas Higher Education Coordinating Board Grant No. ARP 000512-0123-1999 and No. 000512-0063-2001.

\section{References}

[1] W.F. Cheong, S.A. Prahl and A.J. Welch, A review of the optical properties of biological tissues, IEEE J Quantum Elect 26 (1990), 2166-2185.

[2] F.A. Duck, Physical Properties of Tissue, Academic Press Limited, London, 1990.

[3] F.A. Marks, H.W. Tomlinson and G.W. Brooksby, A comprehensive approach to breast cancer detection using light: photon localization by ultrasound modulation and tissue characterization by spectral discrimination, in: Proc SPIE Photon Migration and Imaging in Random Media and Tissues, B. Chance and R.R. Alfano, eds, 1888, 1993, pp. 500-510.

[4] L.-H.V. Wang, S.L. Jacques and X.-M. Zhao, Continuouswave ultrasonic modulation of scattered laser light to image objects in turbid media, Opt Lett 20 (1995), 629-631.

[5] W. Leutz and G. Maret, Ultrasonic modulation of multiply scattered light, Physica B 204 (1995), 14-19.

[6] M. Kempe, M. Larionov, D. Zaslavsky and A.Z. Genack, Acousto-optic tomography with multiply-scattered light, JOpt Soc Am 4 (1997), 1151-1158. 
[7] S. Leveque, A.C. Boccara, M. Lebec and H. Saint-Jalmes, Ultrasonic tagging of photon paths in scattering media: parallel speckle modulation processing, Opt Lett 24 (1999), 181-183.

[8] G. Yao and L.-H.V. Wang, Theoretical and experimental studies of ultrasound-modulated optical tomography in biological tissue, Appl Opt 39 (2000), 659-664.

[9] J. Li and L.-H.V. Wang, Methods for parallel-detectionbased ultrasound-modulated optical tomography, Appl Opt $\mathbf{4 1}$ (2002), 2079-2084.

[10] J. Li, G. Ku and L.-H.V. Wang, Ultrasound-modulated optical tomography of biological tissue using contrast of laser speckles, Appl Opt 41 (2002), 6030-6035.

[11] L.-H.V. Wang and G. Ku, Frequency-swept ultrasoundmodulated optical tomography of scattering media, Opt Lett 23 (1998), 975-977.

[12] G. Yao, S. Jiao and L.-H.V. Wang, Frequency-swept ultrasound-modulated optical tomography in biological tissue by use of parallel detection, Opt Lett 25 (2000), 734-736.

[13] A. Lev, Z. Kotler and B.G. Sfez, Ultrasound tagged light imaging in turbid media in a reflectance geometry, Opt Lett $\mathbf{2 5}$ (2000), 378-380.

[14] S. Leveque-Fort, J. Selb, L. Pottier and A.C. Boccara, In situ local tissue characterization and imaging by backscattering acousto-optic imaging, Opt Commun 196 (2001), 127-131.

[15] E. Granot, A. Lev, Z. Kotler, B.G. Sfez and H. Taitelbaum, Detection of inhomogeneities with ultrasound tagging of light, J Opt Soc Am A 18 (2001), 1962-1967.

[16] A. Lev and B.G. Sfez, Direct, noninvasive detection of photon density in turbid media, Opt Lett 27 (2002), 473-475.

[17] J. Selb, L. Pottier and A.C. Boccara, Nonlinear effects in acousto-optic imaging, Opt Lett 27 (2002), 918-920.

[18] L.-H.V. Wang, Mechanisms of ultrasonic modulation of multiply scattered coherent light: an analytic model, Phys Rev Lett 8704 (2001), 3903-(1-4)

[19] L.-H.V. Wang, Mechanisms of ultrasonic modulation of multiply scattered coherent light: a Monte Carlo model, Opt Lett 26 (2001), 1191-1193.

[20] S. Sakadzic and L.-H.V. Wang, Ultrasonic modulation of multiply scattered coherent light: an analytical model for anisotropically scattering media, Phys Rev E 66 (2002), 026603-(1-9).

[21] M.S. Patterson, B. Chance and B.C. Wilson, Time resolved reflectance and transmittance for the non-invasive measurement of tissue optical properties, Appl Opt 28 (1989), 2331-2336.

[22] M. Atlan, B.C. Forget, F. Ramaz and A.C. Boccara, Private Communication, 2003.

[23] T.A. Whittingham, The safety of ultrasound, Imaging 6 (1994), 33-51.

[24] R.O. Esenaliev, A.A. Karabutov and A.A. Oraevsky, Sensitivity of laser opto-acoustic imaging in detection of small deeply embedded tumors, IEEE J Sel Top Quant 5 (1999), 981-988.

[25] A. Beenen, G. Spanner and R. Niessner, Photo-acoustic depthresolved analysis of tissue models, Appl Spectrosc 51 (1997), 51-57.

[26] C.G.A. Hoelen, F.F.M. de Mul, R. Pongers and A. Dekker, Three-dimensional photo-acoustic imaging of blood vessels in tissue, Opt. Lett. 23 (1998), 648-650.

[27] G. Paltauf and H. Schmidt-Kloiber, Optical method for twodimensional ultrasonic detection, Appl Phys Lett 75 (1999), 1048-1050.

[28] K.P. Kostli, D. Frauchiger, J.J. Niederhauser, G. Paltauf, H.P. Weber and M. Frenz, Opto-acoustic imaging using a threedimensional reconstruction algorithm, IEEE J Sel Top Quant 7 (2001), 918-923.
[29] M.H. Xu, G. Ku and L.-H.V. Wang, Microwave-induced thermo-acoustic tomography using multi-sector scanning, Med Phys 28 (2001), 1958-1963.

[30] R.A. Kruger, P. Liu, Y.R. Fang and C.R. Appledorn, Photoacoustic ultrasound (PAUS)-reconstruction tomography, Med Phys 22 (1995), 1605-1609.

[31] K. Kostli, M. Frenz, H. Bebie and H. Weber, Temporal backward projection of opto-acoustic pressure transients using Fourier transform methods, Phys Med Biol 46 (2001), 18631872.

[32] M. Xu and L.-H.V. Wang, Time-domain reconstruction for thermo-acoustic tomography in a spherical geometry, IEEE Trans Med Imaging 21 (2002), 814-822.

[33] Y. Xu, D. Feng and L.-H.V. Wang, Exact frequency-domain reconstruction for thermo-acoustic tomography: I. Planar geometry, IEEE Trans Med Imaging 21 (2002), 823-828.

[34] Y. Xu, M. Xu, L.-HV. Wang, Exact frequency-domain reconstruction for thermo-acoustic tomography: II. Cylindrical geometry, IEEE Trans Med Imaging 21(7) (2002), 829-833.

[35] G. Ku and L.-H.V. Wang, Scanning electromagnetic-induced thermo-acoustic tomography: signal, resolution, and contrast, Med Phys 28 (2001), 4-10.

[36] J.A. Viator, G. Au, G. Paltauf, S.L. Jacques, S.A. Prahl, H.W. Ren, Z.P. Che and J.S. Nelson, Clinical testing of a photoacoustic probe for port wine stain depth determination, Lasers Surg Med 30 (2002), 141-148.

[37] C.G.A. Hoelen and F.F.M. de Mul, Image reconstruction for photo-acoustic scanning of tissue structures, Appl. Opt. 39 (2000), 5872-5883.

[38] Y.V. Zhulina, Optimal statistical approach to opto-acoustic image reconstruction, Appl. Opt. 39 (2000), 5971-5977.

[39] R.A. Kruger, D.R. Reinecke and G.A. Kruger, Thermoacoustic computed tomography-technical considerations, Med Phys 26 (1999), 1832-1837.

[40] A.C. Tam, Application of photo-acoustic sensing techniques, Rev Mod Phys 58 (1986), 381-431.

[41] V.E. Gusev and A.A. Karabutov, Laser Opto-acoustics, AIP Press, New York, 1993.

[42] M. Xu, Y. Xu and L.-H.V. Wang, Thermo-acoustic imaging in biological tissues: time-domain reconstruction algorithms, IEEE Trans Biomed Eng (2003), accepted.

[43] M. Xu and L.-H. Wang, Formal exact solution and generalized back-projection for an arbitrary recording geometry in photo-acoustic or thermo-acoustic imaging, IEEE Trans Med Imaging (2003), in review.

[44] X. Wang, Y. Xu, M. Xu, S. Yokoo, E.S. Fry and L.-H.V. Wang, Photo-acoustic tomography of biological tissues with high cross-section resolution: Reconstruction and experiment, Med Phys 29 (2002), 2799-2805.

[45] American National Standards Institute, American national standard for the safe use of lasers, Standard Z136.1-2000, ANSI, Inc., New York, NY, 2000

[46] X. Wang, Y. Pang, G. Ku, X. Xie, G. Stoica and L.-H.V. Wang, Non-invasive laser-induced photo-acoustic tomography of the brain in vivo: structural and functional neuroimaging, Nature Biotechnol (2003), accepted.

[47] Y. Pang, X. Wang, G. Ku, G. Stoica and L.-H. Wang, Threedimensional laser-induced photoacoustic tomography of the mouse brain with the skin and skull intact, Opt Lett (2003), in review.

[48] A.A. Oraevesky, A.A. Karabutov, V.S. Solomatin, E.V. Savateeva, V.G. Andreev, Z. Gatalica, H. Singh and R.Y.D. Fleming, Laser optoacoustic imaging of breast cancer in vivo, Proc. SPIE 4256 (2001), 6-15. 
[49] Y. Xu, P. Kuchment and L.-H. Wang, Limited-view thermoacoustic tomography and reconstruction by truncated conjugate gradient, IEEE Trans Med Imaging (2003), accepted with revision.

[50] Y. Xu and L.-H.V. Wang, Effects of acoustic heterogeneity on thermo-acoustic tomography in the breast, IEEE Trans
Ultrason Ferroelectr Freq Control (2003), accepted.

[51] Q. Zhu, T. Durduran, V. Ntziachristos, M. Holboke and A.G. Yodh, Imager that combines near-infrared diffusive light and ultrasound, Opt Lett 24 (1999), 1050-1052.

[52] L.-H.V. Wang and Q. Shen, Sonoluminescent tomography of strongly scattering media, Opt Lett 23 (1998), 561-563. 


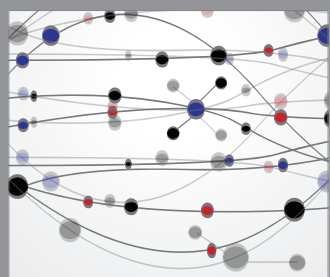

The Scientific World Journal
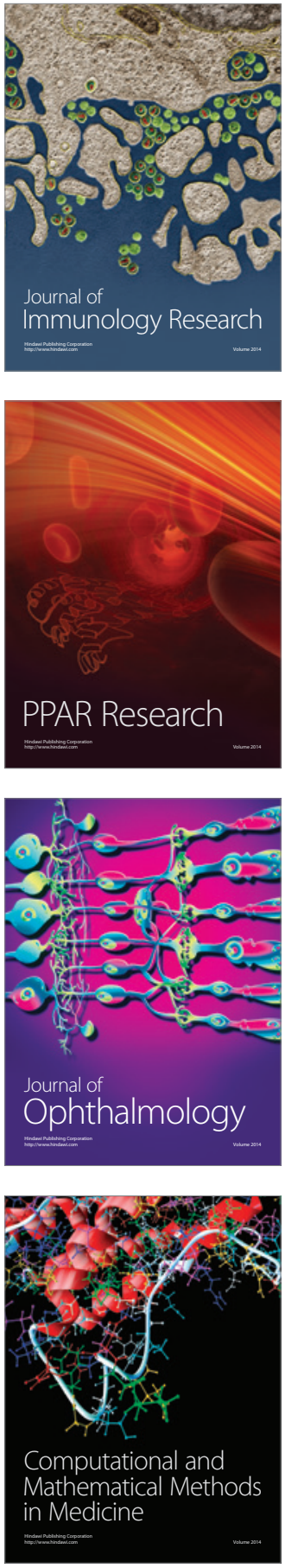

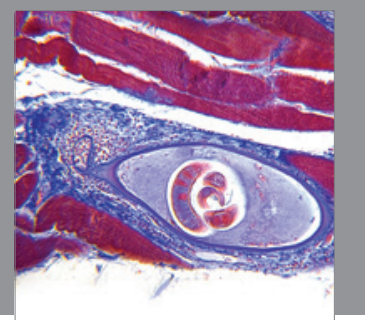

Gastroenterology

Research and Practice
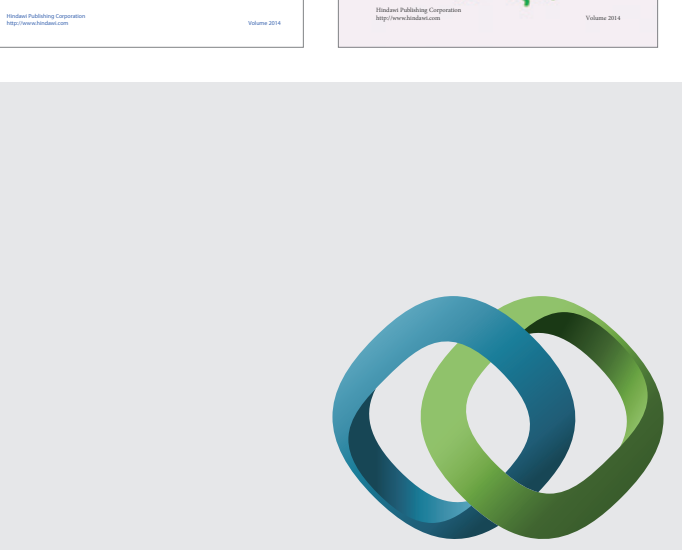

\section{Hindawi}

Submit your manuscripts at

http://www.hindawi.com
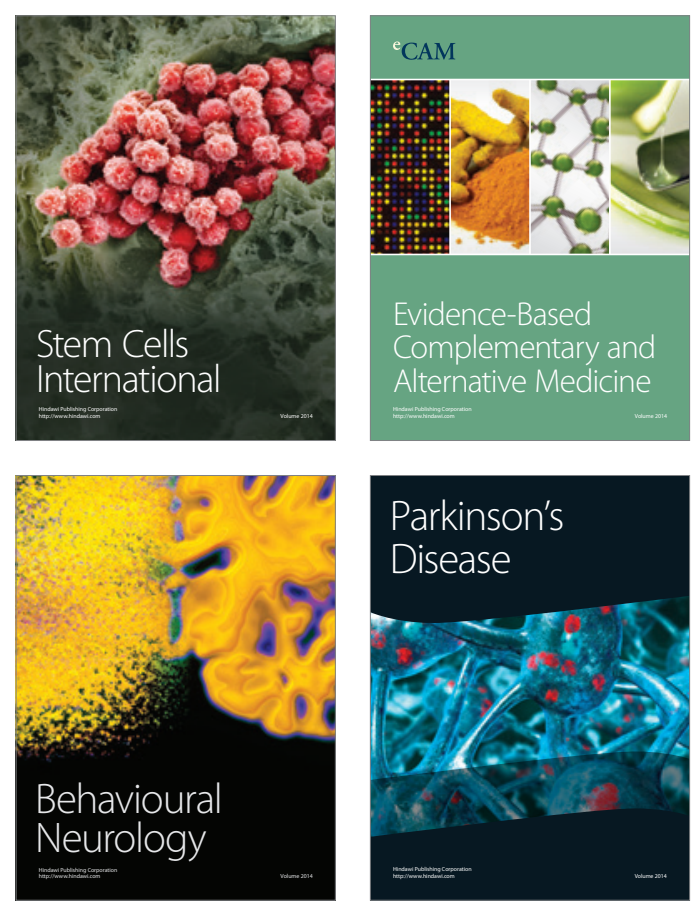

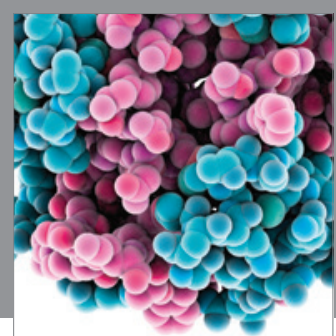

Journal of
Diabetes Research

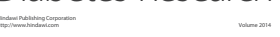

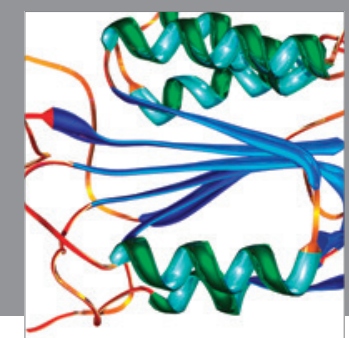

Disease Markers
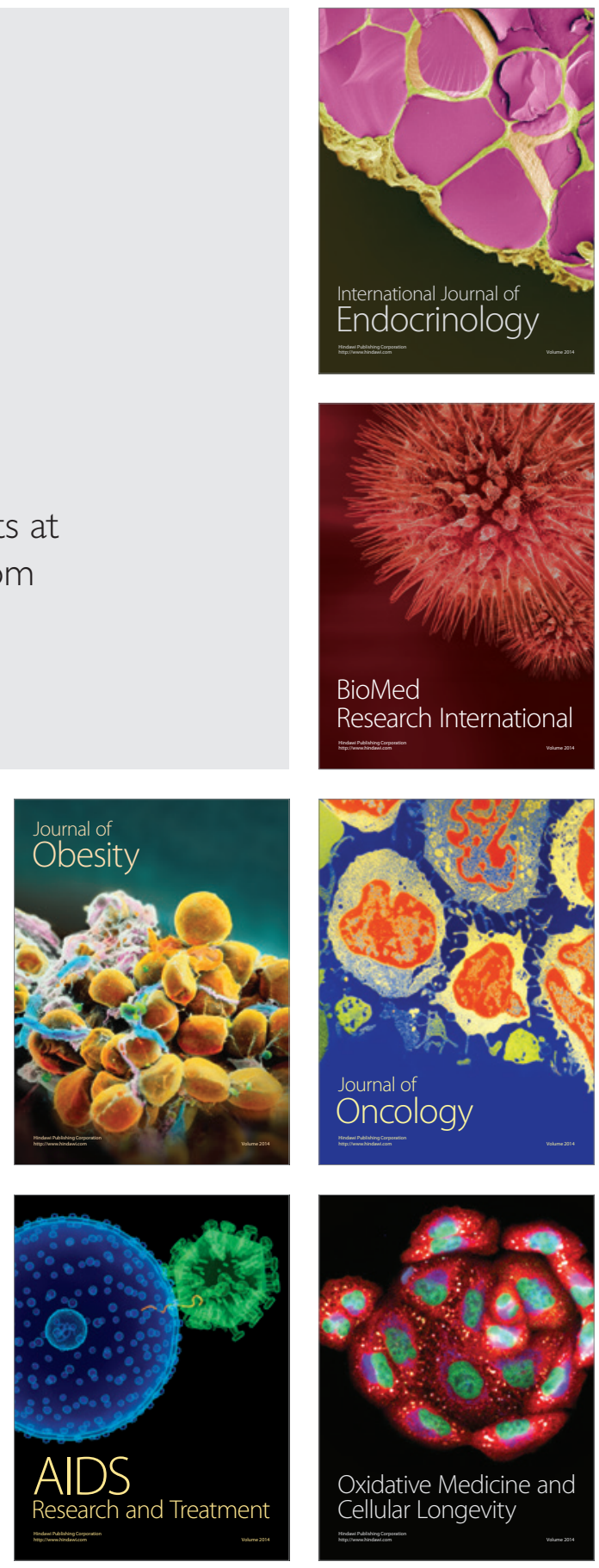